\title{
ARTICLE
}

\section{Underuse of clozapine in treatment-resistant schizophrenia ${ }^{\dagger}$}

\author{
Himanshu Mistry \& David Osborn
}

\begin{abstract}
Himanshu Mistry is an Academic Clinical Fellow at University College London and ST5 in general adult psychiatry. His research interests are treatment-resistant illnesses, psychopharmacotherapy and psychotherapeutic approaches David Osborn is a medical epidemiologist, a senior lecturer in psychiatry at University College London and a consultant psychiatrist. His main interests

are the interface between physical and mental health, psychiatric epidemiology and the provision of effective services for people with severe mental illnesses such as schizophrenia. He works closely with academics in primary care and population health.

Correspondence Dr Himanshu Mistry, Department of Mental Health Sciences, University College London Medical School - Royal Free Hospital Campus, Rowland Hill Street, London NW3 2PF, UK. Email: h.mistry@medsch.ucl.ac.uk
\end{abstract}

${ }^{\dagger}$ See also pp. 256-265, this issue.

\begin{abstract}
SUMMARY
Up to a third of patients with schizophrenia develop treatment resistance. Clozapine has been established as the most effective antipsychotic medication for treatment-resistant schizophrenia. However, it is significantly underutilised in this population. Possible reasons include clinicians' lack of experience with the drug and negative attitudes towards it. African-Caribbean patients are less likely to be treated with clozapine compared with their White counterparts and more likely to have treatment discontinued due to perceived risk of agranulocytosis. This paper discusses the current evidence relating to the underuse of clozapine and factors responsible for this.

\section{DECLARATION OF INTEREST}

None.
\end{abstract}

The term 'treatment-resistant schizophrenia' is broadly used to describe people with a diagnosis of schizophrenia who do not respond adequately to antipsychotic treatment and continue to have residual symptoms and/or poor psychosocial functioning.

Although treatment-resistant schizophrenia is a familiar concept, various definitions and criteria have been proposed over the years to define it as a clinical entity. These include two key concepts: an adequate trial of treatment with antipsychotic medication; and an inadequate response to it. Kane et al (1988) introduced rigorous criteria to define treatment resistance, emphasising persistence of positive symptoms despite receiving 'adequate treatment'. This involved at least three treatment periods of 6 weeks or more with first-generation antipsychotics (FGAs) within the past 5 years at doses equivalent to $1000 \mathrm{mg} /$ day of chlorpromazine. Inadequate response was measured in terms of failure to improve by at least $20 \%$ in total Brief Psychiatric Rating Scale (BPRS) score.

These criteria were later revised in light of emerging evidence, and now the general consensus is that a trial of two antipsychotics including one second-generation antipsychotic (SGA) for 4-6 weeks at a dose equivalent to $400-600 \mathrm{mg}$ of chlorpromazine is an 'adequate trial' (Conley
2001). When evaluating 'adequate response', effect on quality of life is now considered as important as symptom reduction (Lewis 2006).

\section{Prevalence of treatment resistance}

Treatment resistance affects up to $33 \%$ of people with schizophrenia (Essali 2009), although studies using broader definitions of the term suggest that prevalence may be as high as 60\% (Essock 1996).

\section{Efficacy of clozapine in treatment-resistant schizophrenia}

Clozapine is the recommended treatment for treatment-resistant schizophrenia because of robust evidence for its superior efficacy compared with FGAs and other SGAs (Leucht 2009). Kane and colleagues (1988) were the first to establish the efficacy of clozapine over FGAs for strictly defined treatment-resistant schizophrenia in their classic randomised controlled trial, a finding that was subsequently confirmed by other studies (Rosenheck 1997) and meta-analysis (Wahlbeck 1999). More recent studies have reported on the superiority of clozapine over other SGAs on various outcomes such as response to treatment, adherence to medication and patient satisfaction (Lewis 2006; McEvoy 2006). A recent 11-year follow-up study of over 66000 patients with schizophrenia in Finland concluded that antipsychotic treatment was associated with reduced mortality compared with no antipsychotic use and that clozapine was the most effective antipsychotic in this regard (Tiihonen 2009).

The current consensus is that up to $60 \%$ of patients with treatment-resistant schizophrenia will show improvement with clozapine (Meltzer 1997). However, it can take from 6 to 12 months for this to occur (Meltzer 1989). Two to three years of treatment may be required to stabilise symptoms enough to enable patients to return to work and avoid readmissions (Lindstrom 1988; Woodall 2004).

Clozapine has also been established as a cost-effective treatment for treatment-resistant schizophrenia. Studies have reported significant resource savings mainly due to reduced frequency 
of hospital readmissions (Aitchison 1997; Hayhurst 2002). It is estimated that if all patients with treatment-resistant schizophrenia were treated with clozapine, 167 acute beds could be freed in the UK annually. It could potentially prevent 53 suicides and save $£ 8.7$ million in support costs each year (Duggan 2003).

\section{Adverse effects}

The prescription of clozapine is not without risk. About 3\% of patients can develop neutropenia (neutrophil count $0.5-1.5 \times 10^{6} / 1$ ) and $0.8 \%$ agranulocytosis (neutrophil count <0.5 x 106/l); the incidence of fatal agranulocytosis is $0.03 \%$ (Atkin 1996). The risk of both agranulocytosis and neutropenia is highest between 6 and 18 weeks after starting clozapine treatment. However, strict haematological monitoring regimes have reduced mortality significantly (Taylor 2009).

Other important side-effects include myocarditis and myocardiopathy (1 in 1000), pulmonary embolism (1 in 4500), weight gain (more than $20 \%$ patients will gain more than $10 \%$ of baseline weight within a year) and new-onset diabetes mellitus (higher risk in people with a family history of diabetes, male gender or African ethnicity). Additional problems include seizures (3-10\%), orthostatic hypotension (9\%), constipation (10\%), hypersalivation (31-54\%) and drowsiness (1058\%) (Iqbal 2003; Taylor 2009).

\section{Current guidelines}

The clinical benefits of clozapine and its superiority over FGAs and SGAs for treatmentresistant schizophrenia have been accepted by many countries. This is reflected in national guidelines for the treatment of schizophrenia, which advocate for earlier and wider use of clozapine. National Institute for Health and Clinical Excellence (NICE) guidance (National Collaborating Centre for Mental Health 2009) suggests that clozapine should be prescribed after an adequate trial of two antipsychotics (at least one of them being an SGA). Clinical practice guidelines from the Royal Australian and New Zealand College of Psychiatrists (RANZCP) (2005) suggest that clozapine be used early (as soon as treatment resistance has been found to at least two antipsychotics). Incomplete remission, pervasive negative symptoms and suicidality are included as indications. Unlike NICE guidance, the RANZCP guidance does not advocate use of an SGA before switching to clozapine. The American Psychiatric Association (2004) guidelines also recommend clozapine for treatment-resistant schizophrenia and also include suicidality as an indication.

\section{Delay in clozapine initiation}

Despite the wealth of evidence regarding its efficacy and cost-effectiveness, clozapine treatment is often delayed for longer than clinically desirable. In a study of 120 patients conducted in four hospitals in south-east London, Taylor et al (2003) found a mean of 9.2 episodes of antipsychotic prescription before clozapine was initiated. The mean delay in using clozapine was 5 years. High-dose SGAs and polypharmacy are often used instead of clozapine (Fayek 2003).

\section{Variation and underprescribing of clozapine}

In the UK, a 34-fold variation in clozapine prescribing practices has been reported among 12 mental health trusts in the Greater Manchester region over 2 years (Purcell 2000). A subsequent study in the same region (Hayhurst 2003) revealed a reduction to a 16 -fold variation. The main reason for this variation was the different prescribing practices among psychiatrists rather than financial constraints. A study of 45 English mental health trusts found a fivefold variation in clozapine prescribing in 2005-2006 (Downs 2007). It therefore appears that the variation in prescribing clozapine across the UK is slowly reducing.

According to NICE, 63000 individuals had treatment-resistant schizophrenia in 2002 but only 13500 (21\%) patients were receiving clozapine. By 2007 , this figure had risen to 30\% (Downs 2007). In a recent study of clozapine use in assertive outreach teams and rehabilitation units in Hull, UK, Mortimer et al (2010) reported that only 54\% of those with treatment-resistant schizophrenia were receiving clozapine. Underuse of clozapine has also been reported in the USA (Fayek 2003). Clozapine prescription in the USA has been decreasing steadily since the introduction of other SGAs, from 11\% of all SGAs in 1999 to 5\% in 2002 (Kelly 2007a). One study noted that $19 \%$ of all SGA prescriptions in Maryland, USA, were for clozapine compared with $49 \%$ in Victoria, Australia (Conley 2005).

\section{Factors affecting clozapine prescribing}

A telephone survey of 100 psychiatrists in Denmark found that many were reluctant to prescribe clozapine, had little experience in its use and believed that patients treated with clozapine were less satisfied than those treated with other antipsychotics (Nielsen 2010). A survey of consultant psychiatrists working in adolescent units in the UK reported similar concerns. Unfamiliarity with the use of clozapine and apprehension regarding possible serious 
side-effects were prominent factors affecting its use in younger patients (Cirruli 2005).

Although serious side-effects of clozapine are a matter of appropriate concern for clinicians and patients, a tendency on the clinician's part to overestimate the prevalence and severity of sideeffects has been reported (Hodge 2008; Nielsen 2010). In contrast, patients tend not to report major difficulties in taking clozapine. In a study of 570 patients in 27 clozapine clinics in the UK, most patients did not find blood monitoring a significant difficulty and the majority believed that the benefits of clozapine outweighed its potential side-effects (Taylor 2000). Several studies have found that patients report greater improvement and satisfaction with clozapine compared with other antipsychotics (Waserman 2000; Lewis 2006). Beliefs about clozapine differ considerably between patients, their relatives and mental health professionals, as does their evaluation of the positive and negative effects of clozapine treatment. Angermeyer et al (2001) found that both patients and relatives acknowledged the benefits of clozapine over FGAs but they differed in their evaluation of the importance of various side-effects. Patients complained of sideeffects such as hypersalivation, whereas relatives were more concerned about weight gain and clinicians were most concerned about the risk of agranulocytosis.

More aggressive marketing of other SGAs by pharmaceutical companies has also been postulated as a reason for clinicians' reluctance to use clozapine (Kelly 2007a).

\section{Factors affecting continuation of clozapine}

It is estimated that $30 \%$ of patients discontinue clozapine within 6 months and up to $45 \%$ within 2 years (Hayhurst 2002; Whiskey 2003). Longer treatment continuation has been found in hospitals where greater numbers of patients are prescribed clozapine. It may be that clinicians, who have more experience of using clozapine, develop expertise in encouraging patients to continue with clozapine, especially when faced with side-effects in the initial phase of treatment (Whiskey 2003). Patients who complete a year of clozapine treatment have a higher rate of continuation to 2 or more years than those who discontinue treatment at an early stage (Munro 1999). This may be due to the length of time it takes for beneficial effects to become evident. Younger age at initiation of clozapine has consistently been shown to be associated with longer treatment maintenance, whereas the probability of discontinuation increases with advancing age (Munro 1999; Whiskey 2003).

\section{Variability of clozapine prescribing among different ethnic groups}

Patients' ethnicity can influence the choice of drug in the treatment of schizophrenia. Apart from pharmacokinetics specific to different ethnic groups, factors such as culture-specific beliefs about illness, health-related behaviour such as diet and exercise, and societal attitudes can also influence the choice as well as dose of medication prescribed.

It has been well documented that Asian people require lower therapeutic doses of antipsychotic compared with White people and are more likely to experience extrapyramidal side-effects that might affect adherence. South Asians living in Asia who consume a high carbohydrate diet metabolise cytochrome P450 1A2 (CYP1A2) substrates slower and hence require lower doses of antipsychotics than Asians living in the UK who tend to consume diets high in protein, which enhances activity of CYP1A2 (Bhugra 1999; Chaudhury 2008).

Several studies have reported that African American people are less likely to be prescribed an SGA, including clozapine (Kuno 2002; Mark 2002). A more recent study (Mallinger 2006) suggested that African American patients were six times less likely to receive an SGA and half as likely to receive clozapine as White people after controlling for clinical and sociodemographic factors. Possible reasons for this are prescribers' beliefs that African American patients may not adhere to clozapine treatment or to its blood monitoring requirement, or that clinicians are apprehensive about the efficacy of clozapine in Black and minority ethnic groups, which are poorly represented in clinical trials and report a higher prevalence of metabolic complications (Kelly 2006).

Clozapine treatment is also more likely to be discontinued in African-Caribbean patients. This is largely because of pre-existing low neutrophil counts - a condition known as benign ethnic neutropenia (Moeller 1995; Kelly 2007b). Between 25 and $50 \%$ of people of African descent and some ethnic groups in the Middle East have benign ethnic neutropenia (Haddy 1999). It is a normal variant and does not pose a higher risk of infection. African-Caribbean patients with benign ethnic neutropenia are no more at risk of developing agranulocytosis than White patients with normal neutrophil counts (Kerwin 2000). Novartis, the original manufacturer of clozapine, revised its prescribing guidelines in the UK to include special consideration for patients with benign ethnic neutropenia, provided that a haematologist's advice is sought before commencing clozapine (Rajagopal 2005). 


\section{Pharmacogenetics - a new direction?}

There has been a recent surge of interest in research into pharmacogenetic determinants predicting response as well as possible adverse reactions to psychotropic medications. The aim of pharmacogenetic research is to improve the efficacy and safety of drugs prescribed. This move to 'personalised treatment' on the basis of a patient's genetic profile has been seen as a pivotal development. Several review articles have highlighted the developments so far, the potential role of psychopharmacogenetics and future scientific and ethical challenges (Hedgecoe 2006; Arranz 2008). Pharmacogenetics has already been used successfully in other disciplines of medicine but there are several impediments to its implementation in psychiatry owing to the complex interaction of genetic, clinical, environmental and personal variables (De Leon 2009).

Arranz and colleagues (2000) conducted a study in 200 White patients to detect a combination of polymorphisms that would predict response to clozapine. Out of 19 polymorphisms used in the study, they identified 6 single nucleotide polymorphisms (SNPs), which best predicted the response. Schumacher et al (2000) were unable to replicate their findings, partly because of differences in the sample recruited and methodology used.

In 2006, a commercial pharmacogenetic test was launched in the UK that would predict response to clozapine and it is currently being used in several schizophrenia units across the country. The test supports clinical decision-making and encourages patients who are predicted to be responders to continue with clozapine, especially if experiencing poor response or side-effects during the initial phase of treatment. Another such test was launched in the USA in 2007. This claims to predict high (2.5 relative risk) or low ( 0.5 relative risk) risk of agranulocytosis on the basis of analysis of a blood sample and the genotyping of two SNPs of a specific human leukocyte antigen gene (HLADQBI)

\section{KEY POINTS 1}

- As many as $60 \%$ of patients with schizophrenia develop treatment resistance in its broadest definition

- Clozapine is the most effective antipsychotic in reducing mortality in schizophrenia and reducing repeated costly hospital admissions

- Only a third of patients with treatment resistance receive clozapine

- African American patients are half as likely as White patients to receive clozapine
(Kelly 2007a). However, without replication of the findings, the validity of these pharmacogenetic tests is still to be established.

\section{Conclusions}

Treatment resistance occurs in about a third of people with schizophrenia. Clozapine has been consistently established as an effective intervention for treatment-resistant schizophrenia. Starting clozapine at a younger age predicts longer continuation and longer treatment is associated with better clinical outcomes (Key points 1).

However, underuse of clozapine, including delay in initiation and premature discontinuation, has been widely documented. This is particularly common in the African-Caribbean population. Underprescribing of clozapine may be a result of clinicians' limited knowledge and experience in clozapine treatment and overestimation of side-effects. New pharmacogenetic tests that aim to predict responders to clozapine and identify patients more prone to developing agranulocytosis have been developed. However, their validity is not yet established and clinicians' opinions on their usefulness have not been studied to date.

Further studies are required to investigate why a significant number of patients with treatmentresistant schizophrenia in the UK are not prescribed the most effective treatment available and how to address this problem.

\section{References}

Aitchison KJ, Kerwin RW (1997) Cost-effectiveness of clozapine. A UK clinic-based study. British Journal of Psychiatry 171: 125-30.

American Psychiatric Association (2004) Practice Guideline for the Treatment of Patients with Schizophrenia (2nd edn). American Psychiatric Publishing.

Angermeyer M, Loffler W, Muller P, et al (2001) Patients' and relatives' assessment of clozapine treatment. Psychological Medicine 31: 509-17.

Arranz M, Kapur S (2008) Pharmacogenetics in psychiatry: are we ready for widespread clinical use? Schizophrenia Bulletin 34: 1130-44.

Arranz M, Munro J, Birkett J, et al (2000) Pharmacogenetic prediction of clozapine response. Lancet 355: 1615-6.

Atkin K, Kendall F, Gould D, et al (1996) Neutropenia and agranulocytosis in patients receiving clozapine in the UK and Ireland. British Journal of Psychiatry 169: 483-8.

Bhugra D, Bhui K (1999) Ethnic and cultural factors in psychopharmacology. Advances in Psychiatric Treatment 5: 89-95.

Chaudhury IB, Neelam K, Duddu V, et al (2008) Ethnicity and psychopharmacology. Journal of Psychopharmacology 22: 673-80.

Cirulli G (2005) Clozapine prescribing in adolescent psychiatry: survey of prescribing practice in in-patient units. Psychiatric Bulletin 29: 377-80.

Conley R, Kelly D (2001) Management of treatment resistant schizophrenia. Biological Psychiatry 50: 898-911.

Conley R, Kelly D, Lambert T, et al (2005) Comparison of clozapine use in Maryland and in Victoria, Australia. Psychiatric Services 56: 320-3.

De Leon J (2009) Future (or lack of future) of personalised prescription in psychiatry. Pharmacological Research 59: 81-9. 


\section{MCO answers \\ $\begin{array}{lllll}1 \mathrm{~d} & 2 \mathrm{e} & 3 \mathrm{~b} & 4 \mathrm{a} & 5 \mathrm{c}\end{array}$}

Downs J, Zinkler M (2007) Clozapine: national review of postcode prescribing. Psychiatric Bulletin 31: 384-7.

Duggan A, Warner J, Knapp M, et al (2003) Modelling the impact of clozapine on suicide in patients with treatment-resistant schizophrenia in the UK. British Journal of Psychiatry 182: 505-8.

Essali A, Al-Haj Hassan N, Li C, et al (2009) Clozapine versus typical neuroleptic medication for schizophrenia. Cochrane Database of Systematic Reviews 1: CD000059.

Essock S, Hargreaves W, Dohm F, et al (1996) Clozapine eligibility among state hospital patients. Schizophrenia Bulletin 22: 15-25.

Fayek M, Flowers C, Signorelli D, et al (2003) Psychopharmacology: underuse of evidence base treatments in psychiatry. Psychiatric Services 54: $1453-6$.

Haddy T, Rana S, Castro 0 (1999) Benign ethnic neutropenia: what is a normal absolute neutrophil count? Journal of Laboratory and Clinical Medicine 133: 15-22.

Hayhurst K, Brown P, Lewis S (2002) The cost-effectiveness of clozapine: a controlled, population-based mirror-image study. Journal of Psychopharmacology 16: 169-75.

Hayhurst KP, Brown P, Lewis SW (2003) Postcode prescribing for schizophrenia. British Journal of Psychiatry 182: 281-3.

Hedgecoe A (2006) Context, ethics and pharmacogenetics. Studies in History and Philosophy of Biological and Biomedical Sciences 37: 566-82.

Hodge K, Jesperson S (2008) Side effects and treatment with clozapine: a comparison of the views of consumers and their clinicians. International Journal of Mental Health Nursing 17: 2-8.

Iqbal M, Rahman A, Husain Z, et al (2003) Clozapine: a clinical review of adverse effects and management. Annals of Clinical Psychiatry 15 33-48.

Kane J, Honigfeld G, Singer J, et al (1988) Clozapine for the treatmentresistant schizophrenic: a double-blind comparison with chlorpromazine. Archives of General Psychiatry 45: 789-96.

Kelly D, Dixon L, Kreyenbuhl J, et al (2006) Clozapine utilization and outcomes by race in a public mental health system 1994-2000. Journal of Clinical Psychiatry 67: 1404-11.

Kelly D, Kreyenbuhl J, Buchanan R, et al (2007a) Why not clozapine? Clinical Schizophrenia and Related Psychosis 1: 92-5.

Kelly D, Kreyenbuhl J, Dixon L, et al (2007b) Clozapine underutilization and discontinuation in African Americans due to leucopenia. Schizophrenia Bulletin 33: 1221-4.

Kerwin R, Munro J, O'Sullivan D (2000) Paradoxical pattern of haematological risk with clozapine. [Author's reply]. British Journal of Psychiatry 177: 88

Kuno E, Rothbard A (2002) Racial disparities in antipsychotic prescription patterns for patients with schizophrenia. American Journal of Psychiatry 159: $567-72$

Lawson WB (1996) Clinical issues in the pharmacotherapy of AfricanAmericans. Psychopharmacology Bulletin 32: 275-81.

Leucht S, Komossa K, Rummel-Kluge C, et al (2009) A meta-analysis of head to head comparisons of second generation antipsychotics in the treatment of schizophrenia. American Journal of Psychiatry 166: $152-63$.

Lewis S, Barnes T, Davies L, et al (2006) Randomised controlled trial of effect of prescription of clozapine versus other second generation antipsychotic drugs in resistant schizophrenia. Schizophrenia Bulletin 32: 715-23.

Lindstrom LH (1988) The effect of long-term treatment with clozapine in schizophrenia: a retrospective study in 96 patients treated with clozapine for up to 13 years. Acta Psychiatrica Scandinavica 77: 524-9.

Mallinger J, Fisher S, Brown T, et al (2006) Racial disparities in the use of second-generation antipsychotics for the treatment of schizophrenia. Psychiatric Services 57: 133-6.
Mark T, Dirani R, Slade E, et al (2002) Access to new medications to treat schizophrenia. Journal of Behavioral Health Services and Research 29: $15-29$

McEvoy J, Lieberman J, Stroup T, et al (2006) Effectiveness of clozapine versus olanzapine, quetiapine, and risperidone in patients with chronic schizophrenia who did not respond to prior atypical antipsychotic treatment. American Journal of Psychiatry 163: 600-10.

Meltzer H (1997) Treatment resistant schizophrenia - the role of clozapine. Current Medical Research and Opinion 14: 1-20.

Meltzer H, Bastani B, Young Kwon K, et al (1989) A prospective study of clozapine in treatment-resistant schizophrenic patients. Psychopharmacology 99: 68-72.

Moeller F, Chen Y, Steinberg J, et al (1995) Risk factors for clozapine discontinuation among 805 patients in the VA hospital system. Annals of Clinical Psychiatry 7: 167-73

Mortimer AM, Singh P, Shepherd CJ, et al (2010) Clozapine for treatmentresistant schizophrenia: National Institute of Clinical Excellence (NICE) guidance in the real world. Clinical Schizophrenia and Related Psychoses 4: 49-55.

Munro J, O'Sullivan D, Andrews C, et al (1999) Active monitoring of 12,760 clozapine recipients in the UK and Ireland. Beyond pharmacovigilance. British Journal of Psychiatry 175: 576-80.

National Collaborating Centre for Mental Health (2009) Schizophrenia: Core Interventions in Treatment and Management of Schizophrenia in Adults in Primary and Secondary Care (Update). National Institute for Health and Clinical Excellence.

Nielsen J, Dahm M, Lublin H, et al (2010) Psychiatrists' attitude towards and knowledge of clozapine treatment. Journal of Psychopharmacology 24: 965-71.

Purcell H, Lewis S (2000) Postcode prescribing in psychiatry: clozapine in an English county. Psychiatric Bulletin 24: 420-2.

Rajagopal S (2005) Clozapine, agranulocytosis, and benign ethnic neutropenia. Postgraduate Medical Journal 81: 545-6.

Rosenheck R, Cramer J, Xu W, et al (1997) A comparison of clozapine and haloperidol in hospitalized patients with refractory schizophrenia. New England Journal of Medicine 337: 809-15.

Royal Australian and New Zealand College of Psychiatrists (2005) Clinical practice guidelines for the treatment of schizophrenia and related disorders. Australian and New Zealand Journal of Psychiatry 39 : $1-30$

Schumacher J, Schulze TG, Wienker TF, et al (2000) Pharmacogenetic prediction of clozapine response. Lancet 356: 506-7.

Taylor D, Shapland L, Laverick G, et al (2000) Clozapine - a survey of patient perceptions. Psychiatric Bulletin 24: 450-2

Taylor D, Young C, Paton C (2003) Prior antipsychotic prescribing in patients currently receiving clozapine: a case note review. Journal of Clinical Psychiatry 64: 30-4.

Taylor D, Paton C, Kapur S (2009) Clozapine - serious haematological and cardiovascular adverse effects. In The Maudsley Prescribing Guidelines, 10th Edition, 66-7. Informa Healthcare.

Tiihonen J, Lönnqvist J, Wahlbeck K, et al (2009) 11-year follow-up of mortality in patients with schizophrenia: a population-based cohort study (FIN11 study). Lancet 374: 620-7.

Wahlbeck K, Cheine M, Essali A, et al (1999) Evidence of clozapine's effectiveness in schizophrenia: a systematic review and metaanalysis of randomised trials. American Journal of Psychiatry 156: 990-9.

Waserman J, Criollo M (2000) Subjective experiences of clozapine treatment by patients with chronic schizophrenia. Psychiatric Services 51: $666-8$

Whiskey E, Wykes T, Duncan-McConnell D, et al (2003) Continuation of clozapine treatment: practice makes perfect. Psychiatric Bulletin 27: 211-3.

Woodall AA, Menkes DB, Trevelyan TR, et al (2004) A study of clozapine and long-term hospitalisation rates. Psychiatric Bulletin 28 : 285-8. 
MCQs

Select the single best option for each question stem

1 The prevalence of treatment-resistant schizophrenia is accepted to be:

a $1 \%$

b $10 \%$

c $60 \%$

d $33 \%$

e $80 \%$.

2 One of the factors responsible for the underuse of clozapine is:

a evidence-based prescribing by clinicians

b financial constraints at the secondary-care level

c higher rates of non-adherence to clozapine compared with other SGAs

$\mathrm{d}$ risks of clozapine outweighing its benefits e clinician's lack of knowledge and experience in the use of clozapine.

3 Which of the following factors favours longer continuation of clozapine:

a diagnosis of schizophrenia at a young age

b young age at clozapine initiation

c the patient belonging to a Black and minority ethnic group

d history of multiple admissions

e history of extrapyramidal side-effects with previous antipsychotic treatment.

4 Patients with benign ethnic neutropenia are:

a no more likely to develop agranulocytosis on clozapine than are patients with normal neutrophil counts b more susceptible to infection than patients with normal neutrophil counts

c more likely to be White

$\mathrm{d}$ not suitable for clozapine treatment

e less likely to benefit from clozapine compared with other SGAs.

5 The following interventions are used instead of clozapine in routine clinical practice:

a nidotherapy

b other evidence-based pharmacological interventions

c polypharmacy

d psychotherapy

e drug-free trials. 